\title{
Full Duplex Spatial Modulation System Performance Depending on Self-interference Cancellation Accuracy
}

\author{
Yanni Zhou, Florin Hutu, Guillaume Villemaud \\ yanni.zhou@insa-lyon.fr \\ Univ Lyon, INSA Lyon, Inria, CITI, F-69621, Villeurbanne, France
}

\begin{abstract}
Spatial modulation (SM) as a new Multiple-Input Multiple-Output (MIMO) technique is based on transmitting part of the information by activating different emitting antennas. SM increases spectral efficiency and uses only one radio frequency chain. For full-duplex (FD) communication systems, self-interference (SI) is always a central problem. Therefore, combining FD and SM can drastically reduce the difficulty of Self-interference Cancellation (SIC) because of the single SI chain. A Full Duplex Spatial Modulation (FDSM) system is proposed and an active analog SIC is highlighted in this paper. Moreover, the impact of SIC accuracy on the system performance is studied. The results demonstrate that the accuracy requirement will increase as the INR (Self-interference-to-noise Ratio) increases. The FDSM system is less sensitive than the FD system in presence of high estimated error value. Furthermore, an SI detector is presented to resolve the influence of the number of detect symbols.
\end{abstract}

Index Terms-Full Duplex, Spatial Modulation, Selfinterference, Channel Estimation, Bit Error Rate.

\section{INTRODUCTION}

With the development of mobile communication technology, the number of users and data traffic in wireless communications is overgrowing. Moreover, due to the limitation of electromagnetic wave propagation characteristics, the wireless spectrum resources used to support the mobile environment can not be increased endlessly. One of the solutions to overcome this problem is the FD technology. Compared to traditional Frequency Division Duplex (FDD) or Time Division Duplex (TDD), FD can double the spectral efficiency. However, FD will bring strong SI, which severely affects the communication quality. For this reason, SIC has always been the core issue of FD development.

SM was introduced as a new MIMO technology in [1] that uses the active state of antennas as a support for the transmission of information. The superiority of the FDSM system has been theoretically studied in [2][3][4]. Since SM uses a single antenna for the transmission, there is only one data stream of SI for the receiving antenna. The implementation complexity is much lower than that of the multi-stream MIMO. Besides, the spectral efficiency can be improved compared to the FD-SISO (Single-Input SingleOutput) system. As the core difficulty of FD communica- tion, SIC can be divided into passive suppression and active cancellation. Active cancellation includes analog SIC and digital SIC. Passive suppression maximizes the attenuation of the SI signal by adjusting the transmitting/receiving antenna direction or antenna position, coupling, etc. Recent research [5][6] has reduced the strength of SI to an acceptable level by combining the passive suppression and active cancellation, which makes it possible to implement a real FD radio. The key to analog cancellation is SI estimation. More precisely, a signal is transmitted as a cancellation signal, which is inverted with SI in the same frequency. The amplitude and phase of the cancellation signal are adjusted by SI estimation. After SIC in the analog domain, there is still a residual SI, which can be further reduced by using algorithms implemented in digital domain. However, digital SIC is optional. For the single SI channel as in the FDSM system, if SI estimation can be exact, analog cancellation can reduce SI to the noise level. Therefore, the SI estimation results in different accuracy of SIC, which indeed affects the performance of the whole FDSM system.

Previous studies [7][8] analyze the performance of the FD system based on analog cancellation. The performance analysis of the FD system with imperfect SIC has been presented in [9][10]. However, to the authors best knowledge, few publications are available in the literature that discusses the issue of the bit error rate (BER) performance of the FDSM system. The article [11] highlights the better performance of the FDSM system compared with the SM system under imperfect channel estimation. However, it analyzes BER performance by merely defining the constant $\lambda(0 \leq \lambda \leq 1)$ which represents the quality of the channel estimation.

In this paper, we propose a FDSM system over the Rician fading channel with an SI detector. More precisely, Advanced Design System (ADS) - Matlab system co-simulation will be performed in order to study the performance of the proposed system under different accuracies of SIC. Furthermore, the performance of the FDSM system will be compared with that of the traditional FD system.

The remainder of this paper is organized as follows. In Section II, the system is presented in detail. The channel model, the SI, together with the cancellation strategy, are mentioned. Section III shows the ADS-Matlab co-simulation 
results. Section IV summarizes the results of this work, draws conclusions, and points out future research directions.

\section{SYSTEM MODEL}

In this section, we describe a $2 \times 2$ FDSM system over a Rician fading channel with a correction module and a SIC module. Two nodes (namely Node A and Node B) with the same functions are considered. As a FD system, each node has both transmitting and receiving capabilities. The antenna used for emitting or receiving is determined by the input bit stream. The diagram of this system at Node A is presented in Fig.1.

As we can see, the two antennas of node A will be used for transmitting and receiving respectively and simultaneously according to the input bit sequence. The system will be presented in three main parts as follows: Modulation and Demodulation part, Channel model and Channel Correction module part, Self-interference and Cancellation module part.

\section{A. Modulation and Demodulation}

Firstly, the input bit sequence is divided into $\mathrm{N}$ groups. Each group has $\log _{2}\left(N_{t}\right)+\log _{2}(M)$ bits, where $N_{t}$ is the number of antenna at each node, $M$ is the number of distinct symbols that the transmitter is able to send. The first $\log _{2}\left(N_{t}\right)$ bits of each group are used to select the transmitting antenna and the remaining $\log _{2}(M)$ bits are used for the QPSK modulation. We can achieve the desired spectral efficiency by using different modulation methods and configuring different numbers of antennas. In our model, $N_{t}=2, M=4$, hence three bits are transmitted for each output time step. An example is given in Fig.1, the first 3 bits of the input bitstream is 000 . In this case, we active the first antenna for transmitting, and the other is connected to the receiving end. The symbol we transmit is 00 , which is in the first quadrant of the constellation schema.

The demodulation process we are here talking about is the process of recovering the received bit stream after the SIC module and the channel correction module. Since we use QPSK modulation, we have only four possibilities for demodulated symbols. We calculate the minimum distance between the receiving symbol and four possibilities in order to discover the more possibly passing channel and determine the transmitting antenna. A $2 \times 2$ FDSM system has a total of 4 possible channels, where the receiving antenna at each

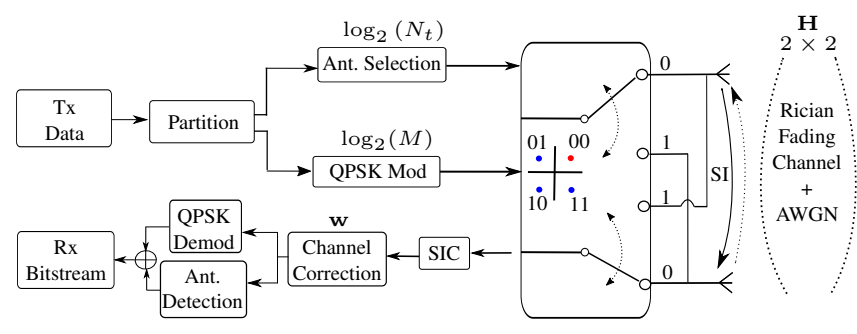

Figure 1. $2 \times 2$ Full Duplex Spatial Modulation System: Node A node occupies two channels. The two possible demodulated corrected symbols will be compared to the four possible symbols to find the minimum difference.

$$
\begin{gathered}
P_{i}=\min \left\{\left|Y_{i}-S_{1}\right|,\left|Y_{i}-S_{2}\right|,\left|Y_{i}-S_{3}\right|,\left|Y_{i}-S_{4}\right|\right\} \\
T=\min \left\{P_{i}\right\} \quad i=1,2
\end{gathered}
$$

Where $S_{1,2,3,4}$ is the QPSK symbol, $Y_{i}$ is the received corrected symbol, $P_{i}$ is the minimal difference between the 4 possible QPSK symbols and the corrected symbol which is transmitted by the $i^{t h}$ antenna. For example, if $\mathrm{T}=P_{1}$, we detect that the first antenna transmits this symbol, and the first bit of this group is 0 .

This detection method has already been applied in a SM system in [12]. However, the complexity is half in this FDSM system compared with that in the SM system. The reason is that only one antenna is used for receiving at each node, which reduces the number of the possible passing channels.

\section{B. Channel model and Channel Correction module}

A time-varying Rician fading channel model $\mathbf{H}$ is considered between two nodes. Additionally, an AWGN noise $\eta$ is considered.

$$
\begin{gathered}
\mathbf{H}=\sqrt{\frac{K}{K+1}} \cdot \mathbf{A}+\sqrt{\frac{1}{K+1}} \cdot \mathbf{V} \\
\mathbf{y}=\mathbf{H} \cdot \mathbf{x}+\boldsymbol{\eta}
\end{gathered}
$$

where $\mathrm{A}$ is a deterministic matrix, $\mathrm{V}$ is a stochastic matrix, $K$ is the Rician factor. More in details, $K$ represents the ratio of the energy in the deterministic Line-of-Sight (LOS) component to the power in the aggregation of the remaining multi-paths.

Our efforts have already focused on the performance of a SM system over this channel model in [12]. It demonstrated that a lower Rician factor does not degrade the performance of the system. Instead, it increases the accuracy of the detection due to the distinct difference between channels, which brings better BER performance. Therefore, a lower Rician factor can be applied under the premise of including a channel correction module.

The function of the channel correction has also been studied. The perfect chanel state information (CSI) can be obtained under the condition that the detection symbols are enough. We already have the experience to build a SM system as well as a FDSM system with a CSI detector. However, in order to more intuitively see the impact of SIC on the system performance, we assume that CSI of the Rician fading channel can be sufficiently obtained for the channel correction module.

\section{Self-interference and Cancellation module}

Here, we define the power ratio of the received SI signal to the receiver thermal noise as INR. The equation that describes the received SI signal $\mathrm{P}_{\mathrm{SI}}$ is as follows:

$$
\mathrm{P}_{\mathrm{SI}}(\mathrm{dBm})=\mathrm{P}_{\mathrm{S}}+\mathrm{INR}-\mathrm{E}_{\mathrm{b}} / \mathrm{N}_{0}
$$

where $\mathrm{P}_{\mathrm{S}}$ is the power of the received signal which is emit from the other node, $\mathrm{E}_{\mathrm{b}} / \mathrm{N}_{0}$ is the signal-to-noise ratio per bit. 
The SI estimation is based on the Least-Squares (LS) algorithm. The specific method is that we shield the transmitting signal from the other node. There is only one self-interference channel at a single node, from the transmitting end to the receiving end, which significantly reduces the channel estimation difficulty. The different numbers of detection symbols result in different accuracy of estimation. We can analyze the relationship between these two factors to select the best amount of detection symbols. According to the LS algorithm, the matrix of SI channel $\mathrm{H}_{\mathrm{SI}}$ is:

$$
\mathrm{H}_{\mathrm{SI}}=\frac{1}{\mathrm{~N}^{2}} \cdot\left[\mathbf{x}^{\mathbf{t}} \cdot \mathbf{x}^{\mathbf{t}^{\mathrm{H}}}\right]^{-1} \cdot \overline{\mathbf{y}^{\mathbf{t}}} \cdot \mathbf{x}^{-1}
$$

where $\mathbf{x}$ is the modulated symbol sent by the transmitting end at each node, $\mathbf{y}$ is the symbol received by the receiving end at the same node, $\mathrm{N}$ is the number of detection symbols. The operator $[\cdot]^{t}$ denotes the transpose, $[\cdot]^{H}$ denotes the Hermitian transpose and $\overline{[\cdot]}$ denotes the complex conjugate.

After estimating the CSI of the SI channel, the amplitude and phase of the SI signal can be estimated. Then, we create a signal with the same amplitude but the inverse phase as the estimated signal to perform the active analog cancellation.

\section{System PERFORMANCE}

A FDSM simulator based on Keysight's ADS and Matlab software is built in order to study the BER performance. At the same time, the FD system is also simulated in order to compare its BER performance with the FDSM system. Three different scenarios will be simulated. Firstly, the effect of SI on system performance without SIC is analyzed. Secondly, we study the impact of the accuracy of SIC in the case of imperfect SI estimation. Finally, a complete FDSM system is constructed by introducing an estimated SI. A time-varying fading channel environment with $K=1$ is considered in simulation. The remaining simulation parameters are gathered in Table I.

\section{A. Without SIC}

The BER performance of the FDSM system and the FD system without SIC are given in Fig.2 and Fig.3. We observe that the BER of the FD system is better than that of the FDSM system, which is normal because of the errors caused by detecting the transmitting antenna. When INR $=-20 \mathrm{~dB}$, the performance of the FDSM system can be basically equivalent to the performance of a $2 \times 2 \mathrm{SM}$ system. The performance of the FD system is theoretically consistent with the performance of a conventional QPSK modulation. When INR $\geq 10 \mathrm{~dB}$, due

Table I

SYSTEM PARAMETERS

\begin{tabular}{cc}
\hline \hline Parameter & Value \\
\hline Bandwidth & $10 \mathrm{MHz}$ \\
Carrier frequency & $2.4 \mathrm{GHz}$ \\
Modulation type & QPSK \\
Data rate & $10 \mathrm{Mbit} / \mathrm{s}$ \\
Total number of input bits & $3 \cdot 10^{5}$ \\
\hline
\end{tabular}

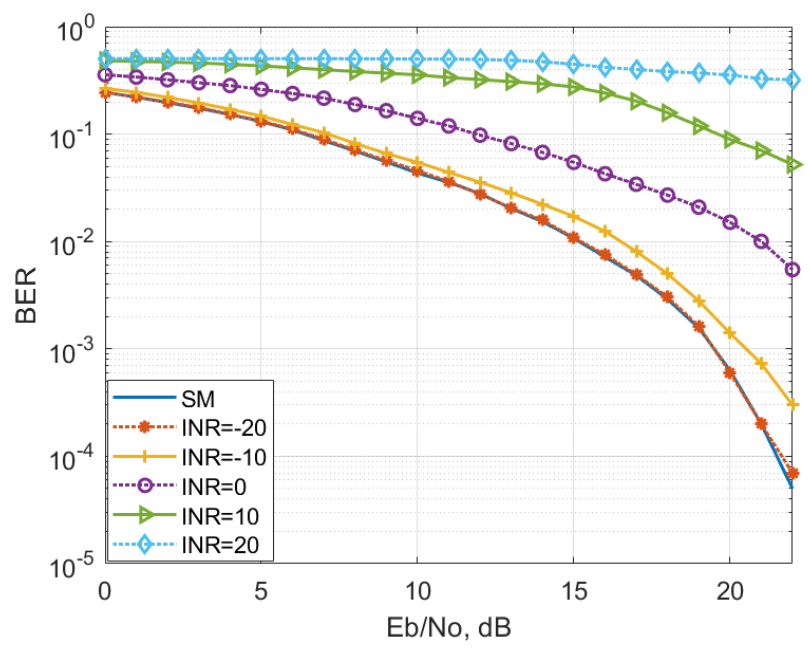

Figure 2. BER performance of the FDSM system without SIC

to the larger SI, the BER of the two systems are maintained at approximately 0.5 while $\mathrm{Eb} / \mathrm{No}$ is at a low level. In addition, we also find that the FD system is more sensitive than the FDSM system. From a poor BER to an acceptable BER, the FD system only needs a small range of Eb/No changes.

\section{B. With different accuracy}

Two factors are defined for evaluating the errors that occur in the SI estimation. The factor $\sigma$ represents the difference of the amplitude between the actual SIC and the perfect SIC, expressed in $\mathrm{dB}$. The factor $\theta$ represents the difference of the phase between the actual SIC and the perfect SIC, expressed in degrees of angle. Fig.4 and Fig.5 present the different accuracies of SIC in terms of the variation of $\sigma$ and $\theta$ for INR $=20,40,60,80$ under $\mathrm{Eb} / \mathrm{No}=10$ ( $\mathrm{dB}$ scale). We emphasize an interesting result in these $3 \mathrm{D}$ figures. As INR increases every $20 \mathrm{~dB}$, we can reduce the $\mathrm{x}$-axis $\mathrm{y}$-axis value range to $10 \%$

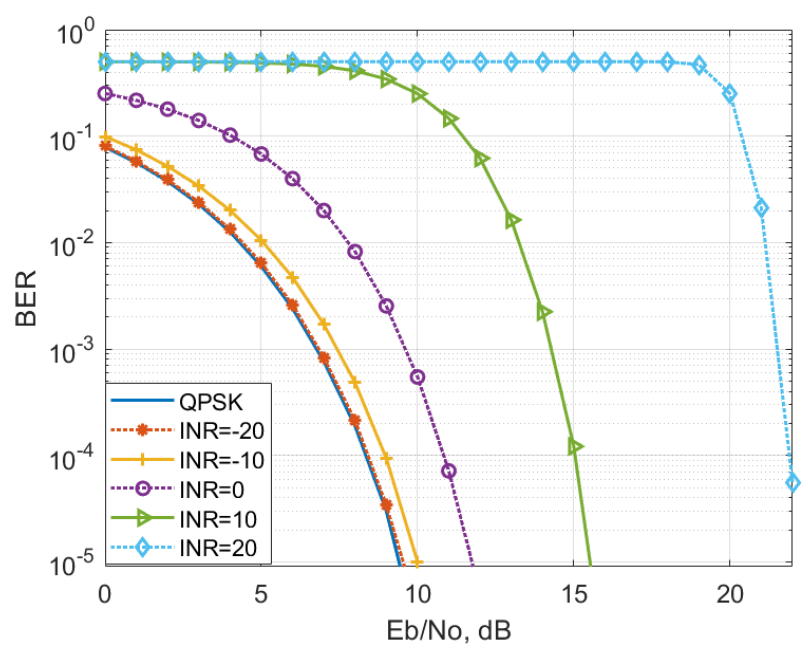

Figure 3. BER performance of the FD system without SIC 

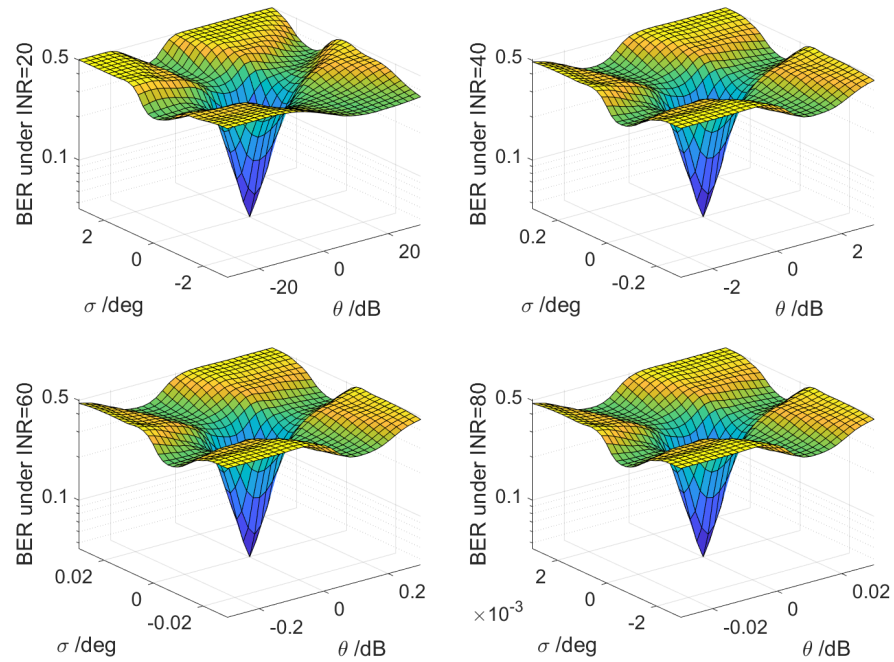

Figure 4. BER performance of the FDSM system with estimation errors for different INRs under $\mathrm{Eb} / \mathrm{No}=10 \mathrm{~dB}$

each time to maintain the $\mathrm{z}$-axis value at the same level. That is to say, when INR increases by $20 \mathrm{~dB}$, the sensitive response to $\sigma$ and $\theta$ of the two systems increases by ten times.

We also note that although the FD system performs better than the FDSM system when there is no error or little error, as $\sigma$ and $\theta$ increase, the BER performance of the FDSM system can reach the same level than the FD system, and even better. Fig.6 shows the difference of the BER value between FDSM system and FD system for $\mathrm{INR}=40 \mathrm{~dB}$ under $\mathrm{Eb} / \mathrm{No}=10 \mathrm{~dB}$. The area with a value greater than zero (yellow area) represents that the BER of the FDSM system is below the BER of the FD system. This finding makes sense because it is impossible to avoid errors in the SI estimation. The FDSM system not only improves the spectral efficiency but also has a performance

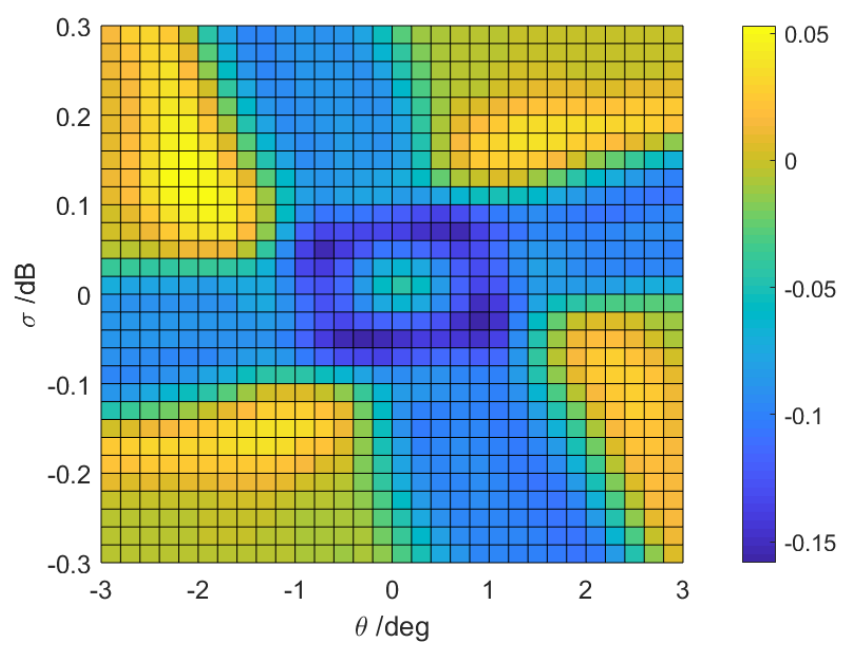

Figure 6. BER difference between FDSM and FD systems for INR=40 dB under $\mathrm{Eb} / \mathrm{No}=10 \mathrm{~dB}$

that is not lower than the FD system as errors increase.

\section{With a real SI detector}

As mentioned in section II, an essential factor affecting the accuracy of SI estimation is the number of the detection symbols. Fig.7 gives the BER performance with real SI estimation for the different INR and the different number of detection symbols under $\mathrm{Eb} / \mathrm{No}=10 \mathrm{~dB}$.

As the INR increases, the BER of the FDSM system is slightly reduced due to the better SI estimation. This result corresponds to our previous conclusions on studying the FD system in [7]. Besides, one can conclude that increasing the number of detection symbols can improve the performance of the system. However, the lower amount of detected symbols

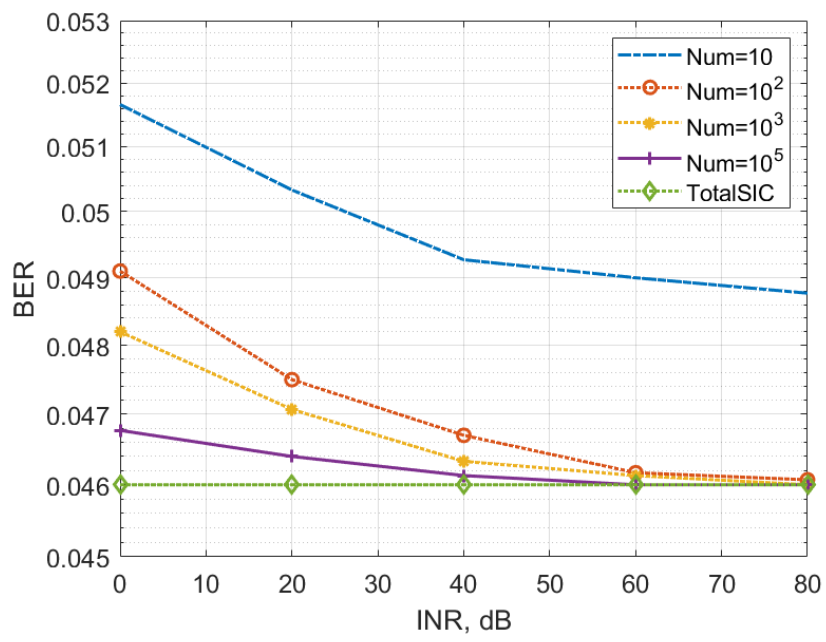

Figure 7. BER performance with SI estimation for different INRs and number
Figure 5. BER performance of the FD system with estimation errors for different INRs under $\mathrm{Eb} / \mathrm{No}=10 \mathrm{~dB}$ of detect symbols under $\mathrm{Eb} / \mathrm{No}=10 \mathrm{~dB}$
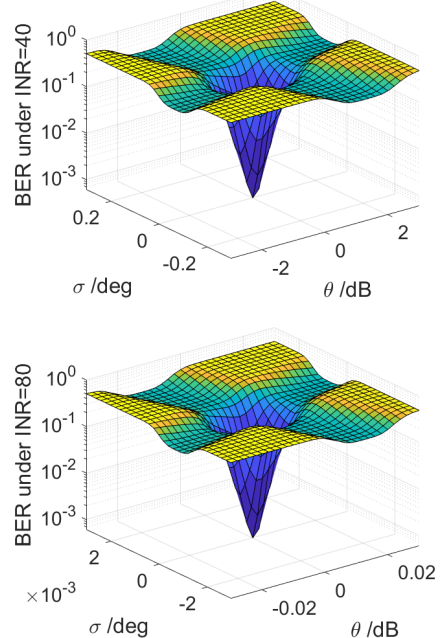
does not make the system perform poorly but at an acceptable level in the case of high INR.

\section{CONCLUSION}

In this paper, a study of a FDSM system is presented. The study was performed in simulation, by using an ADS-Matlab co-simulator. We introduced different modules (antenna selection module, modulation, and demodulation, channel correction module, SI estimation, SIC module, etc.) in order to complete this simulation framework. The impact of SIC accuracy on the BER performance for different INRs was highlighted. We can conclude that an acceptable BER performance needs to maintain the SI estimation error within a small range. The FDSM system performs worse than the FD system when there is no error or are minor errors. However, the lower sensitivity makes the FDSM system maintain a BER performance close to the FD system and even better than the FD system in the case of large errors. Besides, the detector with the different numbers of detection symbols has a lower impact on the performance when INR is large. In other words, it is possible to reduce the number of detection symbols to improve transmission efficiency. Future work will focus on the impact of the receiver non-idealities (for example the local oscillator's phase noise) on the communication quality. Moreover, the development of an experimental setup is envisaged.

\section{REFERENCES}

[1] M. D. Renzo, H. Haas, A. Ghrayeb, S. Sugiura, and L. Hanzo, "Spatial Modulation for Generalized MIMO: Challenges, Opportunities, and Implementation," Proceedings of the IEEE, vol. 102, no. 1, pp. 56 $103,2013$.

[2] B. Jiao, M. Wen, M. Ma, and H. Vincent Poor, "Spatial modulated full duplex," IEEE Wireless Communications Letters, vol. 3, no. 6, pp. 641644, 2014.

[3] J. Zhang, Q. Li, K. J. Kim, Y. Wang, X. Ge, and J. Zhang, "On the Performance of Full-Duplex Two-Way Relay Channels with Spatial Modulation," IEEE Transactions on Communications, vol. 64, no. 12, pp. 4966-4982, dec 2016.

[4] A. Koc, I. Altunbas, and E. Basar, "Two-Way Full-Duplex Spatial Modulation Systems With Wireless Powered AF Relaying," IEEE Wireless Communications Letters, vol. 7, no. 3, pp. 444-447, 2018.

[5] D. Bharadia, E. Mcmilin, and S. Katti, "Full Duplex Radios," Proceedings of ACM SIGCOMM conference, 2013.

[6] A. Sabharwal, P. Schniter, D. Guo, D. W. Bliss, and R. Wichman, "In-Band Full-Duplex Wireless : Challenges and Opportunities," IEEE Journal on Selected Areas in Communications, vol. 32, no. 9, pp. 1637 - 1652, 2014.

[7] Z. Zhan, G. Villemaud, and J.-M. Gorce, "Design and Evaluation of a Wideband Full-Duplex OFDM System Based on AASIC," in IEEE 24th Annual International Symposium on Personal, Indoor, and Mobile Radio Communications (PIMRC), 2013.

[8] - "Analysis and Reduction of the Impact of Thermal Noise on the Full-Duplex OFDM Radio," in IEEE Radio and Wireless Symposium (RWS), 2014.

[9] Z. Zhan, "On Design Concept For Full-Duplex Based Flexible Radio Transceivers," Ph.D. dissertation, INSA de Lyon, 2014

[10] A. Cagatay Cirik, Y. Rong, and Y. Hua, "Achievable Rates of FullDuplex MIMO Radios in Fast Fading Channels with Imperfect Channel Estimation," IEEE Transactions on Signal Processing, vol. 62, no. 15, pp. 3874 - 3886, 2014.

[11] A. Koc, I. Altunbas, and E. Basar, "Full-Duplex Spatial Modulation Systems Under Imperfect Channel State Information," 24th International Conference on Telecommunications (ICT), pp. 1-5, 2017.

[12] Y. Zhou, F. Hutu, and G. Villemaud, "Analysis of a Spatial Modulation System over Time-varying Rician Fading Channel with a CSI Detector," in IEEE Radio and Wireless Symposium (RWS) (in press), 2020. 